\title{
Variant insertion of coracobrachialis muscle in a South Karnataka cadaver
}

\author{
Bhagath K Potu*1, Muddanna S Rao ${ }^{1}$, Soubhagya R Nayak², \\ Venkata R Vollala ${ }^{3}$, Anil K Mandava ${ }^{4}$ and Huban Thomas ${ }^{1}$
} \begin{abstract}
Surgery, Kasturba Hospital, Manipal University, Manipal, Karnataka, India
Email: Bhagath K Potu* - potubidda@gmail.com; Muddanna S Rao - muddannas@yahoo.com; Soubhagya R Nayak - ranjanbhatana@gmail.com; Venkata R Vollala - ramana.anat@gmail.com; Anil K Mandava - mandava_anil2000@yahoo.com; Huban Thomas - huban26@gmail.com

* Corresponding author
\end{abstract}

Address: ${ }^{1}$ Department of Anatomy, Centre for Basic Sciences, Kasturba Medical College, Manipal University, Manipal, Karnataka, India, ${ }^{2}$ Department of Anatomy, Centre for Basic Sciences, Kasturba Medical College, Manipal University, Mangalore, Karnataka, India, ${ }^{3}$ Department of Anatomy, Melaka Manipal Medical College (Manipal Campus), Manipal University, Manipal, Karnataka, India and ${ }^{4}$ Department of Orthopaedic

Published: 31 October 2008

Cases Journal 2008, I:29I doi:I0.1 I86/I757-1626-I-29|

This article is available from: http://www.casesjournal.com/content/I/I/29|

(C) 2008 Potu et al; licensee BioMed Central Ltd.

This is an Open Access article distributed under the terms of the Creative Commons Attribution License (http://creativecommons.org/licenses/by/2.0), which permits unrestricted use, distribution, and reproduction in any medium, provided the original work is properly cited.
Received: 22 October 2008

Accepted: 31 October 2008

\begin{abstract}
Background: The coracobrachialis is a muscle of arm has been known for its morphological variations. The variation of the coracobrachialis reported in this case is unique and to the best of our knowledge this variation is not reported in south karnataka population.

Case presentation: During routine dissection, we observed an unusual insertion of a coracobrachialis muscle with an accessory slip in the right arm of an old male cadaver. It extended from the superficial fibres of coracobrachialis downwards and medially in front of the median nerve and brachial artery and finally inserted on anteromedial aspect of the medial epicondyle.

Conclusion: The existence of abnormal insertion of the coracobrachialis muscle presented in this case should be kept in mind in a patient presenting with high median nerve palsy together with symptoms of brachial artery compression.
\end{abstract}

\section{Background}

Coracobrachialis muscle is classically mentioned as taking its origin from the tip of the coracoid process of the scapula, where it is blended with the medial side of the short head of biceps brachii. The tendon is inserted into the medial border of the shaft of the humerus, where the nutrient foramen is located. The musculocutaneous nerve usually pierces the coracobrachialis muscle in man $[1,2]$.

Morphologic variations of the Coracobrachialis muscle have been known for a long time [3,4] and include accessory slips that attach to the lesser tubercle, medial supra- condylar ridge, medial intermuscular septum. This study describes a variation of the coracobrachialis muscle that crossed anterior to the median nerve and brachial artery in the right arm of an old male cadaver.

\section{Case presentation}

During routine anatomical dissection in our department, we observed an unusual insertion of a coracobrachialis muscle with an accessory slip in the right arm of an old male cadaver. It extended from the superficial fibres of coracobrachialis downwards and medially in front of the median nerve and brachial artery and finally inserted on 


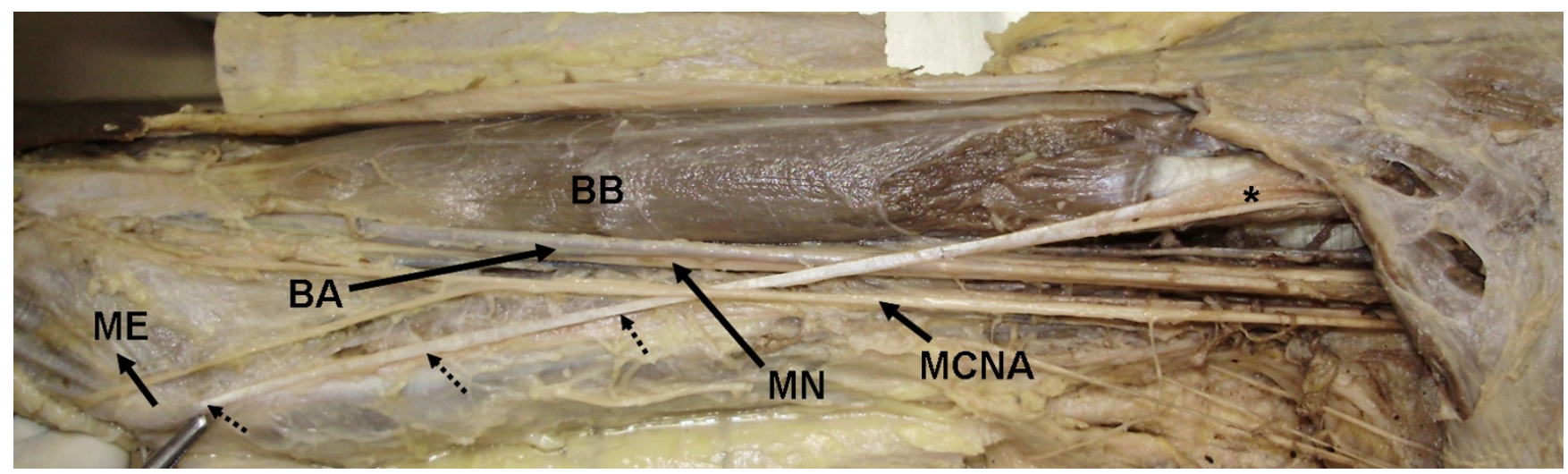

Figure I

A photograph of the right arm of an old male cadaver demonstrating insertion of an accessory slip of the coracobrachialis muscle as a slender tendon (Dashed lines). Notice that: the tendon passing inferiomedially crossing anterior to the Median Nerve (MN) and Brachial artery (BB) and attached to the medial epicondyle (ME) of the humerus. * showing the proximal portion of anomalous tendon. We can also see Biceps brachii muscle (BB) and Medial cutaneous nerve of arm (MCNA) in the picture.

anteromedial aspect of the medial epicondyle (Fig. 1). The length of the part described is $20 \mathrm{cms}$ and the diameter is $0.2 \mathrm{cms}$.

\section{Discussion}

The clinical implication of the accessory slip of coracobrachialis is that it has the potential to cause median nerve entrapment and brachial artery compression. Although we could not detect similar cases described in the available literature, other muscular abnormalities were described, which may lead to a comparable arrangement and injury. Various studies have described the compression of median nerve and brachial artery with anomalous muscles [5-9]. The existence of abnormal insertion of the coracobrachialis muscle should be kept in mind in a patient presenting with high median nerve palsy together with symptoms of brachial artery compression. It may lead to wasting or ischaemic contraction of flexors of the forearm. This variation is important to note during the active use of coracobrachialis as a transposition flap in deformities of infraclavicular and axillary areas and in postmastectomy reconstruction [10], during surgical intervention of the anterior compartment of the arm, such as trauma, tumour, neurovascular disease; while using coracobrachialis as a vascularized muscle for transfer for the treatment of longstanding facial paralysis [11].

The morphological variations of the coracobrachialis muscle may be due to failure of muscle primordia to disappear during embryological development [12] and this might be a reason for the presence of an unusual lengthy accessory insertion of the coracobrachialis muscle reported in this case.

\section{Competing interests}

The authors declare that they have no competing interests.

\section{Authors' contributions}

BKP did the literature search and wrote the case report and also obtained written consent. MSR conceived the study and helped to draft the manuscript. SRN, VRV, AKM and HT helped in the literature search. All authors had gone through the final manuscript and approved it.

\section{Consent}

Written informed consent was obtained from the subject's relative for publication of this case report.

\section{Acknowledgements}

Authors would like to thank Dr. Narga Nair, Professor \& Head of Department of Anatomy, Kasturba Medical College, Manipal University for her support and co-operation. We also thank Dharmavarapu. Praveen Kumar, Postgraduate student in Department of Biochemistry for his help.

\section{References}

I. McMinn RMH, editor: Last's anatomy: regional and applied. 8th edition. Edinburgh: Churchill Livingstone; 1990:79.

2. Williams PL, Warwick R, Dyson M, Bannister LH, editors: Gray's anatomy. 37th edition. Edinburgh-London: Churchill Livingstone; 1989:614-615

3. Wood J: On human muscular variations and their relation to comparative anatomy. J Anat Physiol I867, I:44-59.

4. Howell $A B$, Straus WL: The brachial flexor muscles in primates. Proc US Natl Museum 1932, 80: I-3I.

5. Dharap AS: An anomalous muscle in the distal half of the arm. Surg Radiol Anat 1994, 16:97-99.

6. Nakatani T, Tanaka S, Mizukami S: Bilateral four headed biceps brachii muscles: the median nerve and brachial artery passing through a tunnel formed by a muscle slip from the accessory head. Clin Anat 1998, I I:209-2 I2.

7. El-Naggar M: A study on the morphology of the coracobrachialis muscle and its relationship with the musculocutaneous nerve. Folia Morphol (Warsz) 200I, 60(3):217-224. 
8. EI-Naggar M, AI-Saggaf S: Variant of the Coracobrachialis Muscle With a Tunnel for the Median Nerve and Brachial Artery. Clin Anat 2004, 17:139-143.

9. Vollala VR, Nagabhooshana S, Bhat SM, Potu BK, Gorantla VR: Multiple accessory structures in the upper limb of a single cadaver. Singapore Med J 2008, 49(9):e254-e258.

10. Kopuz C, Icten N, Yildirim M: A rare accessory coracobrachialis muscle: a review of the literature. Surg Radiol Anat 2003, 24:406-10.

II. Taylor GI, Cichowitz A, Ang SG, Seneviratne S, Ashton M: Comparative anatomical study of the gracilis and coracobrachialis muscles: implications for facial reanimation. Plast Reconstr Surg 2003, I I 2:20-30.

12. Grim M: Ultrastructure of the ulnar portion of the contrahent muscle layer in the embryonic human hand. Folia Morphol (Praha) 1972, 20(2): II3-1।5.

Publish with Bio Med Central and every scientist can read your work free of charge

"BioMed Central will be the most significant development for disseminating the results of biomedical research in our lifetime. "

Sir Paul Nurse, Cancer Research UK

Your research papers will be:

- available free of charge to the entire biomedical community

- peer reviewed and published immediately upon acceptance

- cited in PubMed and archived on PubMed Central

- yours - you keep the copyright

Submit your manuscript here:

http://www.biomedcentral.com/info/publishing_adv.asp 